\title{
A simple proof of Debreu's Gap Lemma
}

\author{
Peter Ouwehand* \\ Received: 13 October 2010; Revised: 16 February 2010; Accepted: 23 April 2010
}

\begin{abstract}
Debreu's Gap Lemma is central to the proof of his fundamental result on the existence of continuous utility functions. A short proof based on a standard textbook construction of utility functions on countable linearly ordered sets is presented here. The proof is accessible to students with limited mathematical background, thus making it suitable for inclusion in elementary texts on utility theory.
\end{abstract}

Key words: Utility theory.

\section{Introduction}

In 1954, Gérard Debreu [3] stated conditions for the existence of a continuous utility function. Ten years later, in [4], he noted an error in his original proof, and corrected this error by proving what is now known as the Gap Lemma. In his 1964 paper Debreu [4] introduced the following terminology: "A degenerate set is a set having at most one element. Given a subset $S$ of $\mathbb{R}$, a lacuna of $S$ is a non-degenerate interval of $\mathbb{R}$ without points of $S$, but having a lower bound and an upper bound in $S$; a gap of $S$ is a maximal lacuna of $S . "$ The Gap Lemma may now be stated.

Theorem 1 (Gap Lemma) [4] If $S \subseteq \mathbb{R}$, then there is a strictly increasing function $g: S \rightarrow \mathbb{R}$ such that all the gaps of $g(S)$ are open.

Debreu's proof of Theorem 1 is quite long and complicated — it spans four pages — and is therefore omitted from standard textbooks, such as those by Fishburn [5] and Föllmer and Schied [6]. Nevertheless, the Gap Lemma is a fundamental result in utility theory, and a number of alternative proofs now exist $[1,2,7]$. The aim of this paper is to present another short proof. Though similar in form to the argument provided by Jaffray [7], the proof provided here was constructed independently, and is simpler in execution - compare, for example, the Lemma in $\S 3$ of [7] with Proposition 1 below. However, the main merit of the current proof is didactic: It is based on a standard textbook construction of utility functions on countable linearly ordered sets, and is accessible to students with limited mathematical background, thus making it suitable for inclusion in elementary texts.

${ }^{*}$ Department of Mathematical Sciences, Mathematics Division, University of Stellenbosch, Private Bag X1, Matieland, 7602, South Africa, email: peter_ouwehand@sun.ac.za 


\section{Proof of the Gap Lemma}

Let $(Z, \leq)$ be a countable linearly ordered set, and let $\left\{z_{n}: n \in \mathbb{N}\right\}$ be an enumeration of $Z$ (such that $z_{n}=z_{m}$ only if $n=m$ ). Consider a standard method for defining a utility function (i.e. a strictly increasing function) $f: Z \rightarrow \mathbb{R}$ inductively (see, for example, Kreps [8], Chapter 3): First, let $f\left(z_{0}\right):=\frac{1}{2}$, so that $f\left(z_{0}\right) \in(0,1)$. Next, suppose that $f\left(z_{0}\right), f\left(z_{1}\right), \ldots, f\left(z_{n}\right) \in(0,1)$ have already been defined, and that $f$ is strictly increasing on the set $\left\{z_{0}, z_{1}, \ldots, z_{n}\right\}$. To define $f\left(z_{n+1}\right)$, we consider the following three cases:

Case 1: There exist $j_{0}, j_{1} \leq n$ such that $z_{j_{0}}<z_{n+1}<z_{j_{1}}$. In this case, let

$$
f\left(z_{n+1}\right):=\frac{\max \left\{f\left(z_{j}\right): j \leq n, z_{j}<z_{n+1}\right\}+\min \left\{f\left(z_{j}\right): j \leq n, z_{j}>z_{n+1}\right\}}{2} .
$$

Case 2: $z_{n+1}>\max \left\{z_{j}: j \leq n\right\}$. In this case, let

$$
f\left(z_{n+1}\right):=\frac{\max \left\{f\left(z_{j}\right): j \leq n\right\}+1}{2} .
$$

Case 3: $z_{n+1}<\min \left\{z_{j}: j \leq n\right\}$. In this case, let

$$
f\left(z_{n+1}\right):=\frac{\min \left\{f\left(z_{j}\right): j \leq n\right\}}{2} .
$$

In all cases, $f\left(z_{n+1}\right) \in(0,1)$ also.

This completes the inductive construction of the strictly increasing function $f: Z \rightarrow \mathbb{R}$. Note that the range of $f$ is contained in the unit interval $(0,1)$.

Proposition 1 Let $(Z, \leq)$ be a countable linearly ordered set, and let $f: Z \rightarrow \mathbb{R}$ be the strictly increasing function constructed above. Then $f$ has the following property: If $A, B$ are subsets of $Z$ which satisfy

(i) $A \cup B=Z$,

(ii) $\forall a \in A \forall b \in B[a \leq b]$,

(iii) Either $A$ has no maximum or $B$ has no minimum (or both),

then $\sup _{a \in A} f(a)=\inf _{b \in B} f(b)$.

Proof: Suppose that $A, B$ are subsets of $Z$ satisfying (i), (ii) and (iii). Clearly

$$
\sup _{a \in A} f(a) \leq \inf _{b \in B} f(b)
$$

by (ii). Suppose now that strict inequality holds in (1). We shall obtain a contradiction. Choose $\varepsilon$ so that $0<\varepsilon<\inf _{B} f(b)-\sup _{A} f(a)$. Furthermore, choose $a_{0} \in A$ and $b_{0} \in B$ such that $\sup _{A} f(a)-\varepsilon \leq f\left(a_{0}\right) \leq \sup _{A} f(a)$ and $\inf _{B} f(b) \leq f\left(b_{0}\right) \leq \inf _{B} f(b)+\varepsilon$. Since $a_{0}, b_{0} \in Z$, there are $n, m \in \mathbb{N}$ such that $a_{0}=z_{n}$ and $b_{0}=z_{m}$. If $A$ has no maximum, there must be an element $z_{k} \in A$ such that $z_{k}>a_{0}=z_{n}$ and $k>n, m$. Similarly, if $B$ has 
no minimum, there is an element $z_{k} \in B$ such that $z_{k}<z_{m}=b_{0}$ and $k>n, m$. In either case, we have found an integer $k>n, m$ such that $a_{0}=z_{n}<z_{k}<z_{m}=b_{0}$. Choose $k$ to be the least such integer. By construction of $f$, we must have that $f\left(z_{k}\right)=\frac{f\left(a_{0}\right)+f\left(b_{0}\right)}{2}$. But

$$
\left(\sup _{A} f(a)-\varepsilon\right)+\inf _{B} f(b) \leq f\left(a_{0}\right)+f\left(b_{0}\right) \leq \sup _{A} f(a)+\left(\inf _{B} f(b)+\varepsilon\right)
$$

and thus $f\left(z_{k}\right)$ lies between

$$
\frac{\sup _{A} f(a)+\inf _{B} f(b)}{2}-\frac{\varepsilon}{2} \quad \text { and } \quad \frac{\sup _{A} f(a)+\inf _{B} f(b)}{2}+\frac{\varepsilon}{2} .
$$

By the choice of $\varepsilon$, it is now clear that $\sup _{A} f(a)<f\left(z_{k}\right)<\inf _{B} f(b)$. However, by (i) we see that $z_{k}$ belongs either to $A$ or to $B$, a contradiction.

Before we commence with the proof of the Gap Lemma, we introduce some further terminology. If $(X, \leq)$ is a linear ordering, then a subset $Z \subseteq X$ is said to be order dense if whenever $x<y$ in $X$, there is an element $z \in Z$ such that $x \leq z \leq y$. If $a<b$ in $X$, then we say that $a$ is the predecessor of $b$ (and that $b$ is the successor of $a$ ) in $X$ if the set $\{x \in X: a<x<b\}$ is empty.

Proof of Theorem 1: Let $S \subseteq \mathbb{R}$. It is easy to see that there are at most countably many pairs $a, b \in S$ such that $b$ is a successor of $a$ (in $S$ ), for between any two real numbers there is a rational number, and the set of rational numbers is countable. Thus the set $Z^{\prime}:=\{a \in S: a$ has a successor in $S\}$ is countable. Now let $\left\{I_{n}: n \in \mathbb{N}\right\}$ enumerate all those open intervals with rational endpoints such that $I_{n} \cap S \neq \emptyset$. For each $n$, choose $s_{n} \in I_{n} \cap S$, and let $Z^{\prime \prime}:=\left\{s_{n}: n \in \mathbb{N}\right\}$. Then $Z:=Z^{\prime} \cup Z^{\prime \prime}$ is a countable dense subset of $S$. Construct a utility function $f: Z \rightarrow \mathbb{R}$ as above, and define $g: S \rightarrow \mathbb{R}$ as

$$
g(s):=\sup _{z \in Z, z \leq s} f(z) .
$$

It is clear that $g$ is a utility function, and that $g$ is an extension of $f$.

Let $G$ be a gap in $g(S)$, and let $\ell=\inf G$ and $u=\sup G$. To show that $G$ is open, it suffices to show that $\ell, u \notin G$. Define $A:=\{z \in Z: f(z) \leq \ell\}$ and $B:=\{z \in Z: f(z) \geq u\}$. Because $G$ is a gap, properties (i) and (ii) of Proposition 1 are satisfied. Since

$$
\sup _{z \in A} f(z) \leq \ell<u \leq \inf _{z \in B} f(z)
$$

property (iii) must fail, so that $A$ has a maximum element $a_{0}$, and $B$ a minimum element $b_{0}$. Because $G$ is a gap (i.e. a maximal lacuna), it is clear that $f\left(a_{0}\right)=\ell$ and $f\left(b_{0}\right)=u$. Thus $\ell, u \in g(S)$, i.e. $\ell, u \notin G$.

\section{Final remarks}

In the proof of the Gap Lemma, we defined

$$
g(s)=\sup _{z \in Z, z \leq s} f(z),
$$


where $Z$ is a countable dense subset of $S$ which contains all predecessor-successor pairs. We claim also that

$$
g(s)=\inf _{z \in Z, z \geq s} f(z) .
$$

For let $A=\{z \in Z: z \leq s\}$ and let $B=\{z \in Z: z \geq s\}$. Then properties (i) and (ii) of Proposition 1 are satisfied. Thus, if

$$
\sup _{z \in A} f(z)<\inf _{z \in B} f(z)
$$

then property (iii) must fail, i.e. $A$ must have a maximum $a_{0}$, and $B$ must have a minimum $b_{0}$. We cannot have $a_{0}=b_{0}$; so, by properties (i) and (ii), $b_{0}$ is a successor of $a_{0}$ (in $S$ ), which implies that $a_{0}, b_{0} \in Z$. Since $a_{0} \leq s \leq b_{0}$, we see that $s \in Z$, which immediately yields

$$
\sup _{z \leq s} f(z)=f(x)=\inf _{z \geq s} f(z)
$$

a contradiction.

Furthermore, from (2) it follows that the map $g: S \rightarrow \mathbb{R}$ is continuous if $(S, \leq)$ is endowed with its order topology.

\section{Acknowledgement}

The author would like to thank one of the anonymous referees for bringing the paper by Jaffray [7] to his attention.

\section{References}

[1] Beardon AF, 1992, Debreu's Gap Theorem, Economic Theory, 2, pp. 150-152.

[2] Bosi G \& Menta GB, 2002, Existence of a semicontinuous or continuous utility function: A unified approach and an elementary proof, Journal of Mathematical Economics, 38, pp. 311-328.

[3] Debreu G, 1954, Representation of a preference ordering by a numerical function, pp. 159-165 in Thrall R, Coombs CC \& Davis R (Eds), Decision processes, Wiley, San Francisco (CA).

[4] Debreu G, 1964, Continuity properties of paretian utility, International Economic Review, 5, pp. 285-293.

[5] Fishburn PC, 1970, Utility theory for decision making, John Wiley and Sons, San Francisco (CA).

[6] Föllmer H \& Schied A, 2002, Stochastic finance, Walter de Gruyter, Berlin.

[7] JAFFray J, 1975, Existence of a continuous utility function: An elementary proof, Econometrica, 43, pp. 981-983.

[8] Kreps D, 1988, Notes on the theory of choice, Westview Press, Boulder (CO). 